\title{
Discriminación Contra Las Mujeres En El Estado De Hidalgo, México: Una Revisión De La Última Encuesta Nacional Sobre Discriminación
}

\author{
Carlos Mejía Reyes (PhD) \\ Karina Pizarro Hernández (PhD) \\ Adrián Galindo Castro (PhD) \\ Instituto de Ciencias Sociales y Humanidades (ICSHU), \\ Universidad Autónoma del Estado de Hidalgo, México
}

Doi: 10.19044/esj.2017.v13n35p161 URL:http://dx.doi.org/10.19044/esj.2017.v13n35p161

\begin{abstract}
Discrimination against women is a structural phenomenon that international organizations have tried to eradicate through different legal instruments. In this regard, Mexico has signed several treaties and despite such initiatives, the discriminatory practice continues to diminish the fundamental rights of half of the population. Based on the interests of the state of Hidalgo (Mexico), this social phenomenon is regarded to be very crucial. The treatment of the subject is carried out by means of a quantitative review, and by comparing the last National Survey on Discrimination of the sub-sample of the State of Hidalgo with the national results. The analysis of the situation in a federative entity allows us to approach, in a diagnostic way, a phenomenon that is approached a little by the social sciences of the context. This research is the product of the "Diagnosis of discrimination in Pachuca de Soto, Hidalgo, and conurbada zone" project, and it was funded by the PROMEP of the Undersecretary of Higher Education.
\end{abstract}

Keywords: Discrimination, Women, Hidalgo state, ENADIS 2010

\section{Resumen}

La discriminación contra las mujeres se ha conformado como un fenómeno estructural que los organismos internacionales han intentado erradicar mediante instrumentos legales diversos. México ha firmado varios tratados al respecto y a pesar de tales iniciativas, la práctica discriminatoria continua mermando los derechos fundamentales de la mitad de la población. Particularmente, este fenómeno social, en el contexto que nos interesa, el estado de Hidalgo (México), la situación se ha definido como grave. El tratamiento del tema se lleva a cabo mediante una revisión cuantitativa 
analizando comparativamente la última Encuesta Nacional sobre Discriminación, de la sub-muestra del Estado de Hidalgo con los resultados nacionales. Así, el análisis de la situación en una entidad federativa permite aproximarnos de forma diagnóstica a un fenómeno poco abordado por las ciencias sociales del contexto. Esta investigación es producto del proyecto "Diagnóstico de la discriminación en Pachuca de Soto, Hidalgo y zona conurbada" financiado por el PROMEP de la Subsecretaría de Educación Superior.

Palabras clave: Discriminación, mujeres, Estado de Hidalgo, ENADIS 2010

\section{Introducción}

Es en la Carta de las Naciones Unidas de 1945 que México firma la adhesión a un tratado internacional en el que reconoce por vez primera los derechos fundamentales e igualdad de derechos entre hombres y mujeres. En tal documento se especifica y legitima la manifestación de que las mujeres han sido discriminadas de forma histórica y estructural, por tal razón se han limitado la igualdad de derechos. Esto se concretó en 1948 con la Declaración Universal de los Derechos Humanos. De esta emanó La Comisión Jurídica y Social de la Mujer, instancia instructora del Consejo de las Naciones Unidas (González de Pazos, 1989).

Una instancia que precedió tal enunciación fue la Organización Internacional del Trabajo (OIT), Con este antecedente la ONU (Organización de las Naciones Unidas) generó la Convención Internacional para la Supresión del tráfico de Mujeres y niños en 1921, ratificada por México hasta 1932. Posteriormente en 1933 firmó otra referente al tráfico de mujeres adultas.

Ante tal andamiaje jurídico el estado mexicano ha implantado diversas herramientas legales para intentar abatir la discriminación, sin embargo la aplicación de los mandatos es altamente desfavorable para la lucha contra la discriminación en los imaginarios sociales generales y particularmente en el Estado de Hidalgo.

El estado de Hidalgo es uno de los treinta dos que conforma la república mexicana. Cuenta con 64 municipios. Su capital es el municipio de Pachuca de Soto. En el territorio se localizan tres principales etnias: Nahuas, Otomíes y Tepehuas. Existe como idioma oficial el español, pero se usan nueve lenguas más: Nahua, Otomí, Tepehua, Mixteco, Zapoteco, Totonaca, Mazahua, Maya y Huasteco. Su principal actividad económica es el comercio, construcción, transporte y la industria alimentaria.

Para el año 2010 contó con una población de 2665018 habitantes en una superficie de $20813 \mathrm{~km}$ cuadrados. De los cuales el $51.7 \%$ son hombres y el $48.3 \%$ son mujeres. Que representa el 2.4\% de la población nacional. 
Se conforma básicamente por población joven en el $50 \%$ del total. La esperanza de vida es de 73.6 años de edad (Fernández, 2014).

Se ubicó como el sexto estado del país con mayores índices de marginación y posee un alto índice de migración internacional, muy por encima de la media nacional.

\section{Legislaciones Internacionales y Mexicanas Contra la Discriminación de las Mujeres y Niñas}

México, hasta el año de 1981 es que ratificó la firma del convenio internacional CEDAW (Convención Internacional sobre la Eliminación de todas las formas de Discriminación contra la Mujer) que la ONU propuso dos años antes. En este documento se obliga al Estado mexicano logar la equidad de género y empoderamiento de las mujeres y niñas, además de incorporar la perspectiva de género en el total de instituciones y acciones para garantizar igualdad así como también la erradicación de la discriminación directa e indirecta contra las mujeres (CEDAW, 2011).

En esta convención se define en el primer artículo que:

"A los efectos de la presente Convención, la expresión "discriminación contra la mujer" denotará toda distinción, exclusión o restricción basada en el sexo que tenga por objeto $\mathrm{o}$ por resultado menoscabar o anular el reconocimiento, goce o ejercicio por la mujer, independientemente de su estado civil, sobre la base de la igualdad del hombre y la mujer, de los derechos humanos y las libertades fundamentales en las esferas política, económica, social, cultural y civil o en cualquier otra esfera." (CEDAW, 2011)

Ante la obligación internacional, la máxima carta legal de México ha mantenido y ratificado en el artículo primero de la Constitución Política de los Estados Unidos Mexicanos (2016) que:

"Queda prohibida toda discriminación motivada por origen étnico o nacional, el género, la edad, las discapacidades, la condición social, las condiciones de salud, la religión, las opiniones, las preferencias sexuales, el estado civil o cualquier otra que atente contra la dignidad humana y tenga por objeto anular o menoscabar los derechos y libertades de las personas."

De este párrafo se derivó la creación, en 2003, de la Ley Federal para Prevenir y Eliminar la Discriminación (LFPED) que, a su vez, en su artículo 16 establece la generación del Consejo Nacional para Prevenir y Eliminar la Discriminación (CONAPRED). 
Este organismo público y descentralizado, define discriminación como:

“ $\{\ldots\}$ toda distinción, exclusión, restricción o preferencia que, por acción u omisión, con intención o sin ella, no sea objetiva, racional ni proporcional y tenga por objeto o resultado obstaculizar, restringir, impedir, menoscabar o anular el reconocimiento, goce o ejercicio de los derechos humanos y libertades, cuando se base en uno o más de los siguientes motivos: el origen étnico o nacional,el color de piel, la cultura, el sexo, el género, la edad, las discapacidades, la condición social, económica, de salud o jurídica, la religión, la apariencia física, las características genéticas, la situación migratoria, el embarazo, la lengua, las opiniones, las preferencias sexuales, la identidad o filiación política, el estado civil, la situación familiar, las responsabilidades familiares, el idioma, los antecedentes penales o cualquier otro motivo; También se entenderá como discriminación la homofobia, misoginia, cualquier manifestación de xenofobia, segregación racial, antisemitismo, así como la discriminación racial y otras formas conexas de intolerancia." (LFPED, 2014)

De tal forma que, establecer políticas públicas concretas para identificar este fenómeno social y definirlo como "problemática social" (Araiza, 2005), conforma una paso firme reconocer e intervenir con fines de suprimir prácticas que laceran los derechos humanos fundamentales así como el tejido social. Reconocimiento, tardío por cierto, que México hizo de esta problemática ante la negación sistemática del "problema" (Rincón, 2005).

Con fines de perfeccionar tal legislación, el Estado Mexicano se propuso modificar la LFPED en 2014 para establecer responsabilidades puntuales a la CONAPRED de recibir y atender quejas ciudadanas e imponer medidas administrativas y/o reparadoras a servidores(as) públicos o particulares que lesionen los derechos antidiscriminatorios. También estableció políticas positivas o acciones afirmativas para generar procesos de igualdad de trato para grupos en situaciones desventajosas. Esto, en atención al Plan Nacional de Desarrollo 2013-2018 que el gobierno federal se planteó, que obedece a uno de los Objetivos de Desarrollo del Milenio de la ONU fijados en 2015.

\section{Algunos Datos Sobre Discriminación en México}

Sin embargo, ante todo este andamiaje jurídico e institucional, las prácticas discriminatorias no dejan de hacerse visibles, al grado de que se 
reflejan en las propias encuestas de discriminación que la CONAPRED elabora desde 2005. En esta primera, los resultados expusieron datos sumamente reveladores; ya que nueve de cada diez mujeres, indígenas, personas con discapacidad, adultos(as) mayores, personas con credos religiosos "minoritarios" y no heterosexuales opinaron que existe discriminación por su condición. Y uno(a) de cada tres personas de estos grupos refirió haber sufrido discriminación de forma reciente. Casi 5 de cada 10 informantes señaló que no estarían dispuestas a permitir que en su casa viviera una persona homosexual. Cuatro de cada diez señaló que las personas de origen indígena siempre tendrán limitaciones sociales por sus características "raciales" y esa misma proporción de encuestados refirió que estaría dispuesto a organizarse para impedir que grupos de indígenas se establezcan cerca de su residencia. También uno de cada tres señaló que para salir de la pobreza es necesario dejar de comportarse como indígena.

Con respecto a las mujeres, cuya discriminación es estructural e histórica (Serret, 2001; Lagarde, 2012; Rubín, 2003; Bourdieu, 2005; Oakley, 1977), la encuesta mostró que uno de cada cuatro pediría un examen de embarazo a una mujer al solicitar empleo. También uno de cada cuatro asume que las mujeres son agredidas sexualmente porque son ellas quienes provocan a los hombres. Además uno de cada tres informantes opinó que es "normal" que las mujeres obtengan menos salario que los varones. Con esto, el 94\% de las mujeres encuestadas señaló que la discriminación contra las mujeres es patente y siete de cada diez refirió que los espacios donde se sufre más discriminación es en la familia y el empleo (ENADIS, 2005).

La segunda edición de esta encuesta, elaborada en 2010, ampliada en temáticas no consideradas en la primera, muestra algunos resultados relevantes como que seis de cada diez creen que la riqueza es una de las principales razones que provocan divisiones entre la gente. Cuatro de cada diez lo señaló con respecto a las preferencias sexuales, ideas políticas, valores y religión. Con respecto a la etnia lo refirieron tres de cada diez.

De igual manera cuatro de cada diez refirió no estar dispuesto a permitir que en su hogar vivan personas homosexuales ni lesbianas. Dos de diez lo refirió con personas de "otra raza", cultura, lengua y nacionalidad. De igual forma se reconoce, en tres de cada diez encuestados(as), que no se respetan los derechos de migrantes provenientes de Centroamérica; y en cuatro de diez con personas indígenas. Existe la opinión en cuatro de diez encuestados(as) que se trata de forma diferenciada en función del tono de piel "morena".

De manera extendida se mostró que los sectores también discriminados son los y las jóvenes, personas adultas mayores, migrantes y con alguna discapacidad; primordialmente para acceder a empleos (ENADIS, 2011). 
A pesar de los esfuerzos institucionales para mitigar la exclusión social, los datos indican que ha tenido tendencias contrarias ya que se ha registrado un aumento de $17 \%$ en casos atendidos de 2014 a 2015 . Del total de denuncias en este último año, el $64 \%$ fueron actos cometidos por particulares, mayoritariamente por actos de discriminación en función de la apariencia física, discapacidad y embarazo. El resto de denuncias atribuidas a servidores públicos tienen como motivo la condición de salud, discapacidad y género (Méndez, 2016). En el mismo año, otra estudio cuantitativo señaló que la mitad de la población encuestada ha sufrido discriminación por su apariencia física y acento; y que cuatro de cada diez asegura que las razones de la discriminación se atribuyen a sus costumbres, cultura y color de piel (Redacción Animal Político, 2015).

\section{Discriminación Contra las Mujeres. Una Aproximación Cuantitativa Desde la Encuesta Nacional Sobre Discriminación (ENADIS, 2010) Metodología}

Una forma de conocer lo que opinan "las que sufren y han padecido históricamente las consecuencias directas de la discriminación” (Bucio \& Fix-Fierro, 2011) es mediante ejercicios de levantamiento de encuestas a nivel nacional. En este caso utilizaremos la ENADIS 2010 de forma comparativa entre los resultados de la sub-muestra del Estado de Hidalgo en comparación del promedio nacional. Con ello se pretende aproximarnos al tema particular en dos niveles: la discriminación contra un sector social que representa la mitad de la población de una entidad y por otro confrontar la distancia entre los resultados nacionales con el caso específico.

Esta encuesta fue levantada en 2010 mediante un cuestionario general aplicado a hogares y otro de manera específica a algún integrante familiar aleatoriamente elegido con la finalidad de recuperar sus valores y actitudes referentes a la discriminación. También se realizaron diez cuestionarios dirigidos a grupos vulnerables determinados, previamente delimitados y otro individual a personas vulnerables (mujeres, niñas y niños, jóvenes, adultos mayores, migrantes, "minorías" religiosas, étnicas, culturales y sexuales, personas trabajadoras del hogar u con alguna capacidad diferente). Así, la muestra seleccionada consistió en 13751 hogares y 52095 personas de las 32 entidades federativas y en 31 municipios (CONAPRED, 2011; Barba, 2012).

Para la submuestra del Estado de Hidalgo, se utilizaron los resultados de 688 informantes. De los cuales el $51.5 \%$ son mujeres y el restante $48.5 \%$ son varones. La media de edad es de 32 años. El porcentaje de informantes sin instrucción educativa es de 4.8\%, el 65.4\% tienen educación básica, $20.7 \%$ tiene educación media y el $8.5 \%$ con educación profesional. El $55.1 \%$ es empleado mientras que el resto, $44.9 \%$, no labora.

Para el tratamiento de esta fuente de datos, utilizaremos el método ya 
que tiene s como fundamento metodológico que trasciende la simple observación de sentido común y tiene por objetivo un ordenado y persistente modus de analizar semejanzas/diferencias para extraer conclusiones (Colino, 2009).

Los objetivos puntuales del método comparativo pueden ser numerados en cuatro principales o fundamentales. Por un lado es mantener una mirada libre de prejuicios inherentes a la pertenencia cultural o social desde el cuál se evalúan procesos sociales; es decir romper la mirada etnocéntrica (Durkheim, 2001; Colino, 2009). El siguiente es de carácter descriptivo y consiste en comprender de mejor manera los procesos o situaciones o fenómenos sociales a estudiar en cada caso para dar cuenta de sus especificidades (Mills, Van de Bunt \& De Brujin, 2006). El tercer objetivo es de tipo explicativo, en el sentido de ordenar o clasificar para generar tipologías de los hechos observados (Ragin \& Zaret, 1983). Por último la comparación se orienta regularmente a la generalización a partir de regularidades sociales y hallazgo de factores principales con fines de generar un modelo explicativo y testar hipótesis (Vigour, 2011; Kohn, 1987; Rihoux, 2006).

Para la realización de este ejercicio se aludieron a una serie de parámetros mínimos que hicieron del ejercicio analítico uno adecuado a las circunstancias. Entre los criterios básicos consideraron fueron una serie de conceptos empíricos comparables, es decir tener claridad con lo que es o no confrontable. Por otra parte establecer variables comparables.; con esto se procede al análisis solamente a fenómenos sociales de la misma clase; o sea analizar principios comunes en contextos diferentes (Sartori, 1970; Rodríguez, 2011; Pennings, Keman \& Kleinnijenhuis, 2006; Caïs, 2002).

\section{Resultados}

Así, la primer variable a examinar es acerca de un tópico básico que implica consideraciones de violencia pública contra las mujeres (Grafica 1). 
Gráfica 1. Porcentaje de acuerdo o no que mujeres provocan a hombres.

Comparativo submuestra Hidalgo y muestra México

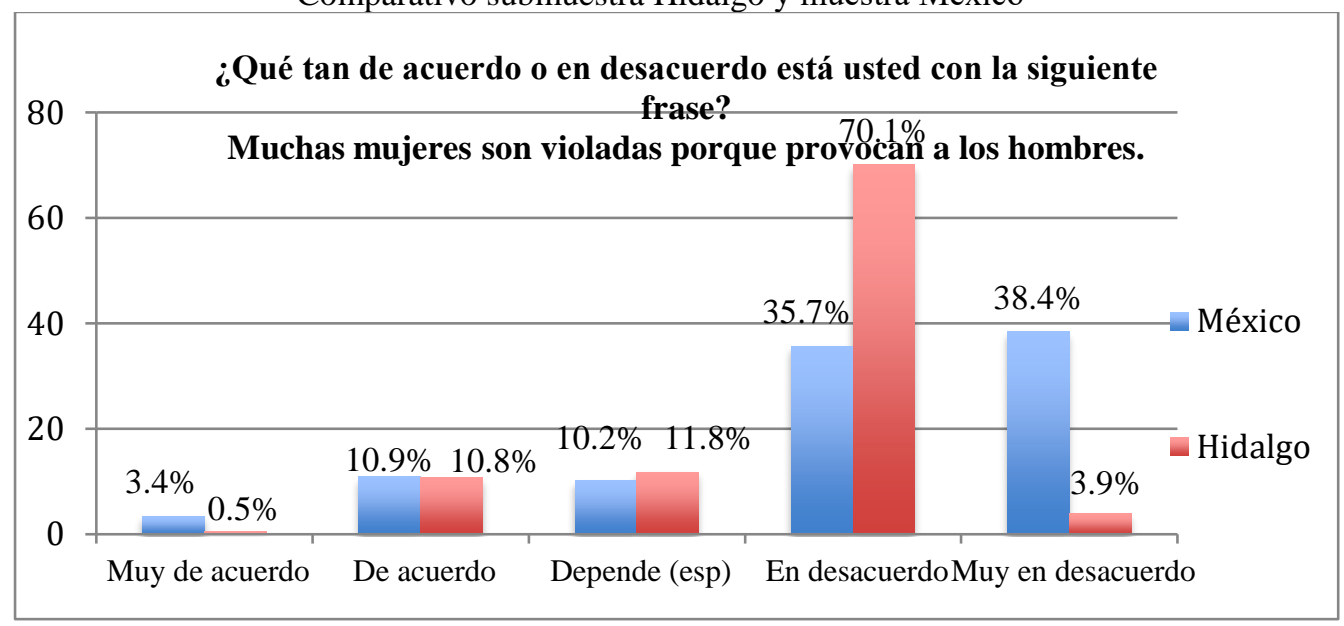

Fuente: Elaboración propia con base en ENADIS 2010.

Podemos apreciar que el desacuerdo es más pronunciado en la submuestra Hidalguense en comparación a la muestra nacional. Sin embargo, y lo que resulta importante referir es que para los y las hidalguenses, en un punto porcentual más, consideran que "depende" las circunstancias, las mujeres son las que provocan a los hombres.

El siguiente tema refiere a la percepción acerca la interrupción del embarazo y la aceptación o reprobación del derecho de decisión que las mujeres tienen de su cuerpo (Gráfica 2).

Gráfica 2. Porcentaje de aceptación del aborto.

Comparativo submuestra Hidalgo y muestra México

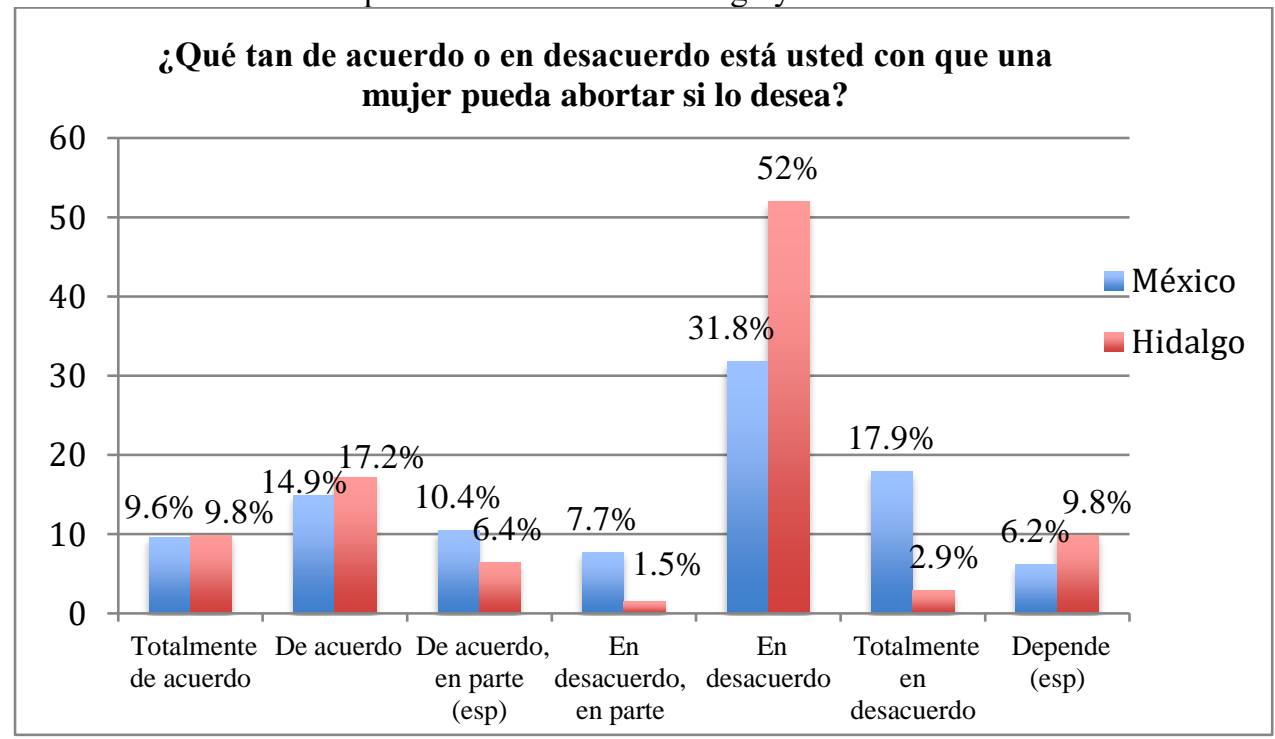

Fuente: Elaboración propia con base en ENDAIS 2010. 
En comparación nacional, Hidalgo posee un porcentaje ligeramente más alto de informantes que consideran estar de acuerdo con que una mujer pueda abortar, pero el porcentaje aumenta considerablemente en poco más de la mitad de informantes que lo reprueban categóricamente. Esto en niveles altamente superiores al porcentaje del país. Lo cual permite aseverar que esta entidad puede ser catalogada como conservadora al respecto al derecho de decidir de las mujeres sobre su cuerpo.

Subsiguientemente, los imaginarios referentes hacia la concepción del respeto de las mujeres (Gráfica 3) de igual manera reflejan las distancias considerables entre el promedio nacional y el caso de la entidad; esto porque solamente $23.5 \%$ de hidalguenses consideraron que se respetan los derechos de las mujeres, mientras que el porcentaje nacional se ubica con 32.3\%. Dejando ver que se considera que en la entidad se respetan en menor medida tales derechos. Lo anterior se replica en la respuesta negativa porque el 29.4\% de las personas encuestadas en Hidalgo considera que definitivamente no se respetan los derechos de las mujeres en contraste del $25 \%$ a nivel país.

Gráfica 3. Porcentaje percepción de respeto de derechos de la mujeres.

Comparativo submuestra Hidalgo y muestra México

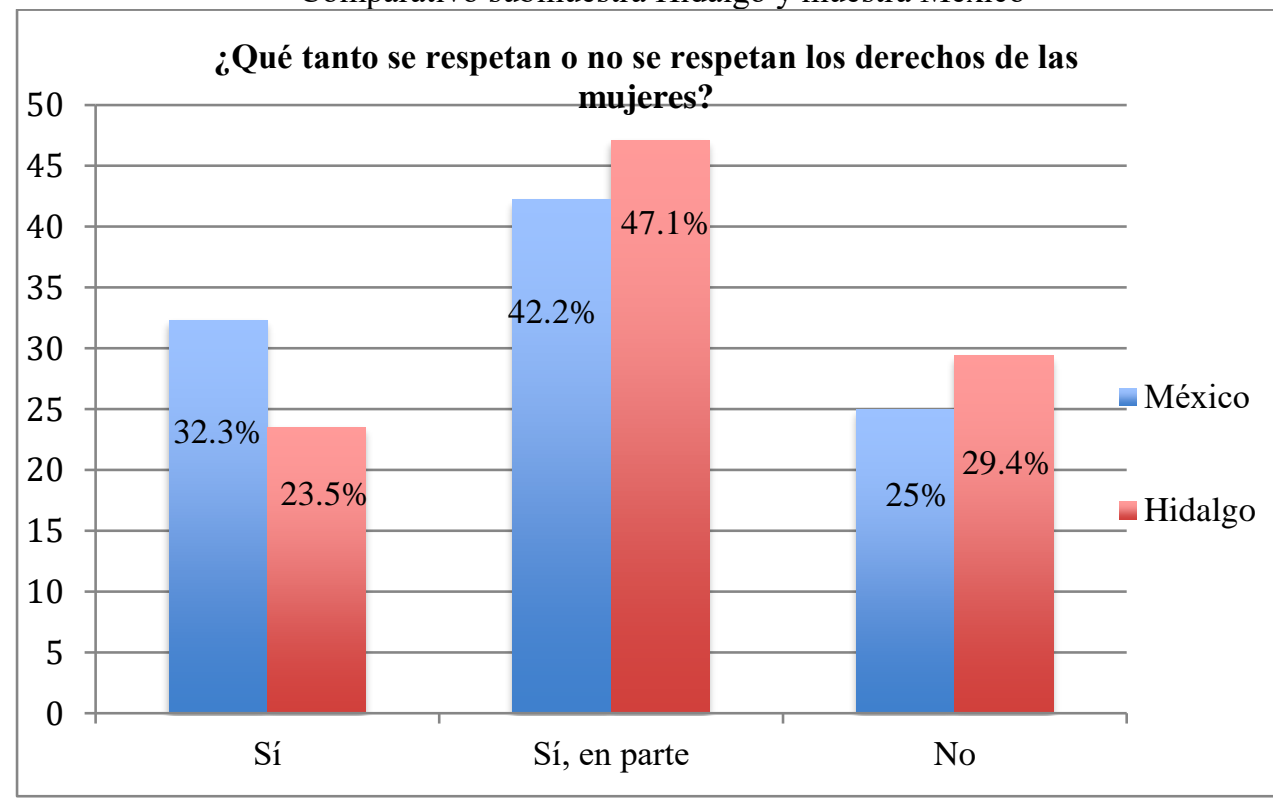

Fuente: Elaboración propia con base ENADIS 2010.

De tal manera que en Hidalgo es mayoritariamente considerado el irrespeto por los derechos de las mujeres. También en la Gráfica 4 se refleja la valoración en las mujeres, específicamente, que no se respetan los derechos por razones exclusivamente de género; a diferencia de los varones. 
Gráfica 4. Porcentaje percepción de derechos no respetados entre hombres y mujeres.

Comparativo submuestra Hidalgo y muestra México

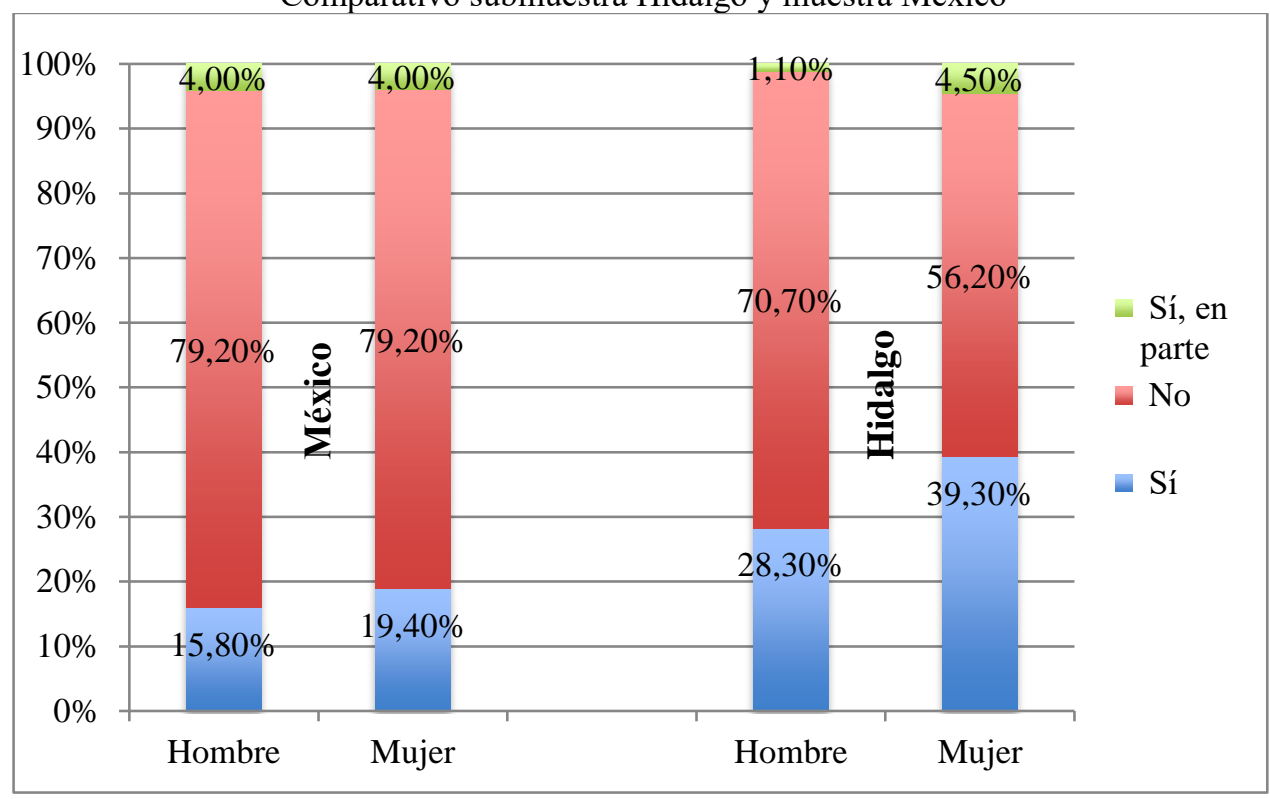

Fuente: Elaboración propia con base en ENADIS 2010.

El 39.3\% de las mujeres hidalguenses encuestadas han sentido que sus derechos no han sido respetados, más de diez puntos por encima de los hombres en el mismo estdo de la república, más del doble de los hombres a nivel país y veinte puntos porcentuales por arriba del promedio nacional. Dejando ver por lo tanto que 4 de cada 10 mujeres de la entidad ha sentido vulnerables sus derechos; el doble que en la encuesta general.

De forma abierta, cuando se cuestionó a las mujeres informantes cuál es el principal problema que consideran les aqueja, las respuestas fueron diversas (Gráfica 5). 
Gráfica 5. Porcentaje percepción del principal problema para las mujeres. Muestra México

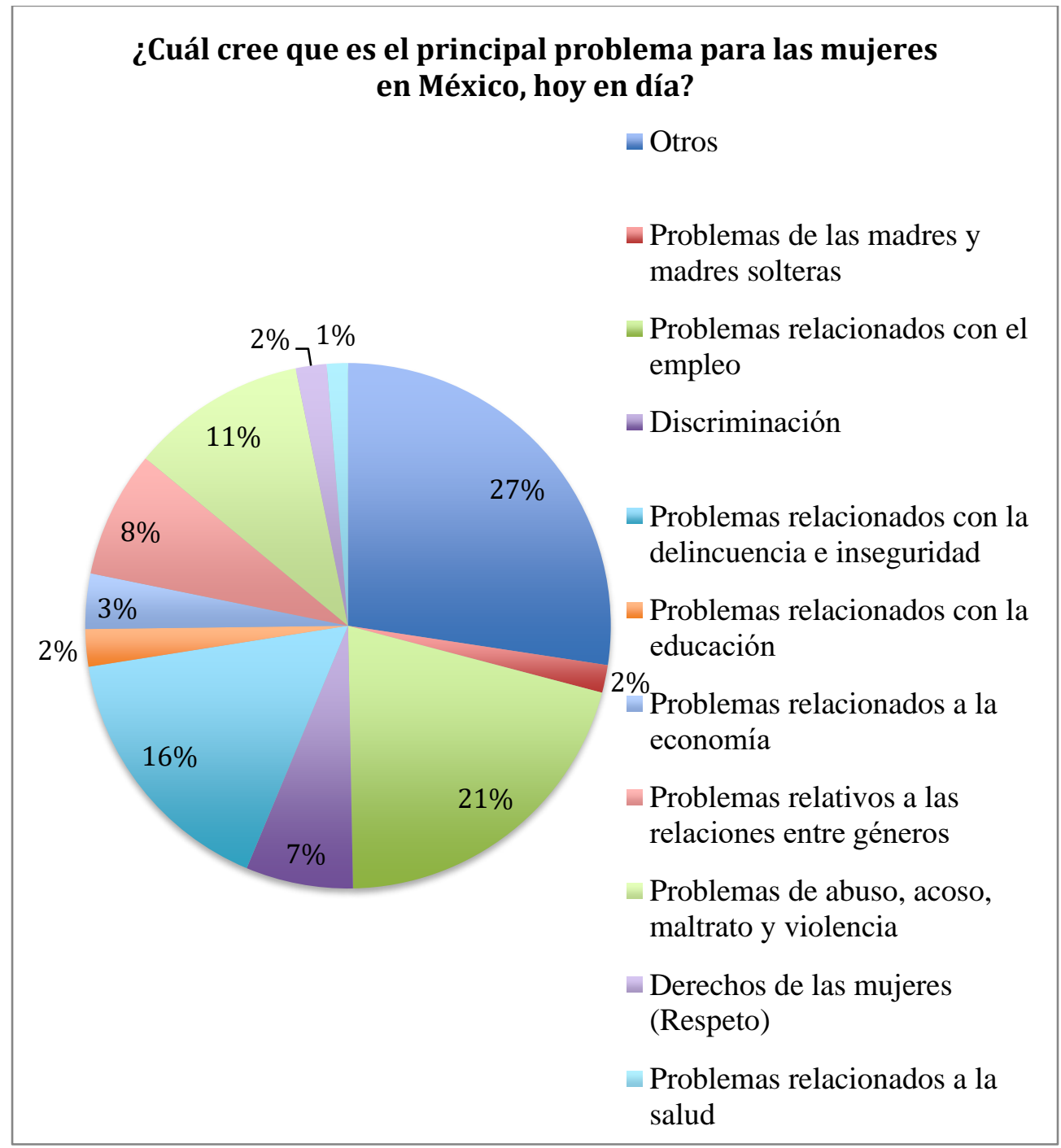

Fuente: Elaboración propia con base en ENADIS 2010.

Los resultados permiten observar que para este sector social, el "problema" que mayoritariamente fue mencionado fue el empleo y los relacionados con ello (20.6\%), enseguida los problemas inherentes a la inseguridad pública (16.2\%), en tercer lugar los relacionados a la violencia sexual y acoso callejero, violencia y maltrato (10.8\%), después las relativas a las relaciones de géneros $(7.6 \%)$, y discriminación $(6.6 \%)$. 
Gráfica 6. Porcentaje percepción del principal problema para las mujeres.

Submuestra Hidalgo

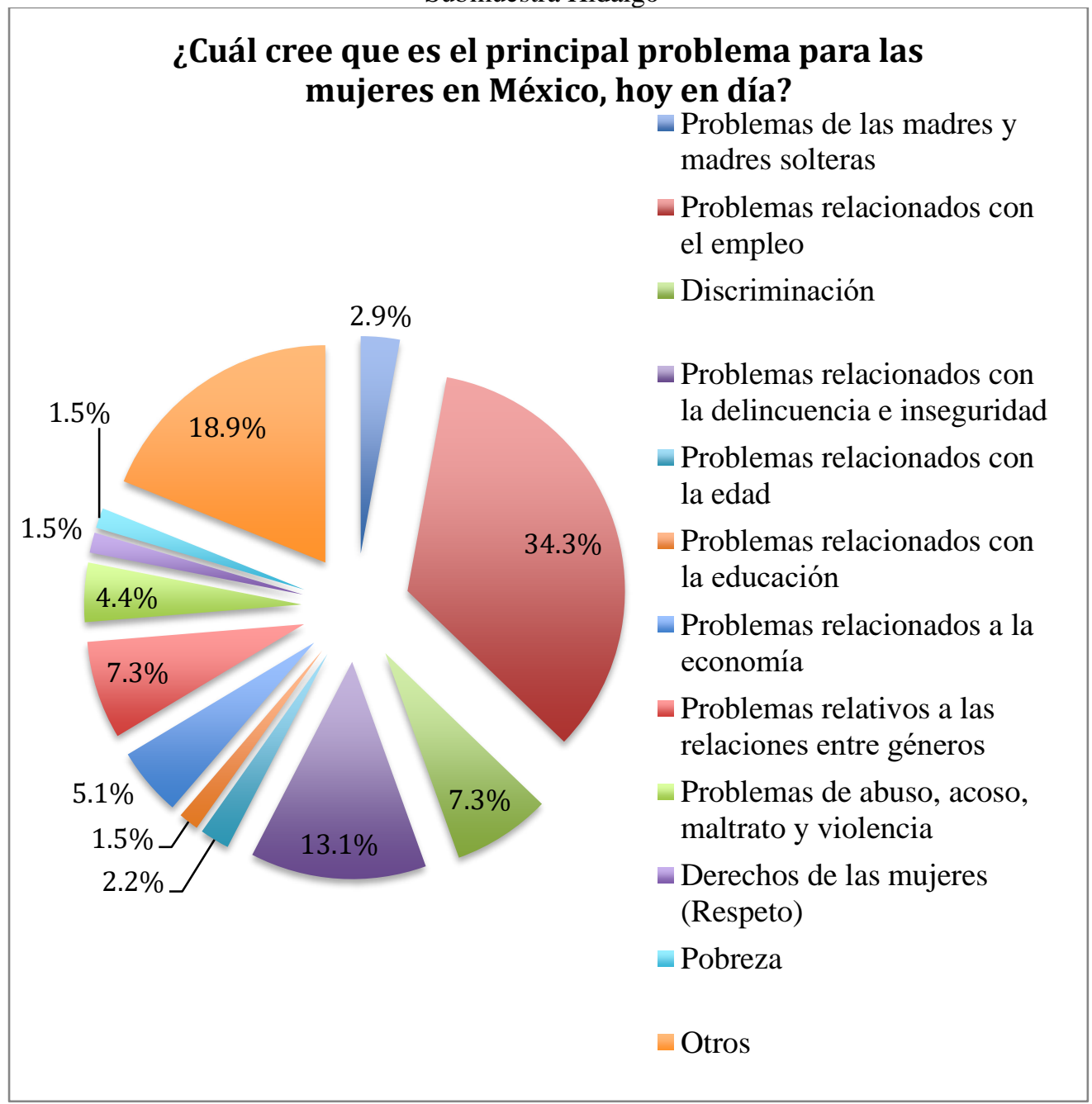

Fuente: Elaboración propia con base ENADIS 2010.

El la Gráfica 6 muestra el caso Hidalguense, en el que las entrevistadas refieren que los principales problemas para las mujeres son los relativos al empleo (34.3\%), delincuencia (13.1\%), discriminación y de relaciones entre géneros (7.3\%), de la economía (5.1\%), de abuso o maltrato y violencia (4.4\%), de madres solteras (2.9\%), edad (2.2\%). Con lo anterior apreciamos que es en las mujeres de esta entidad se señala la discriminación como la tercera opción más referida, mientras que en el promedio nacional lo es en quinta preferencia.

Aunado a lo anterior, imaginariamente las mujeres Hidalguense legitiman aún la predominancia económica en los varones con respecto a los salarios en el núcleo familiar (Gráfica 7). 
Gráfica 7. Porcentaje percepción de ingresos entre hombres y mujeres.

Comparativo submuestra Hidalgo y muestra México

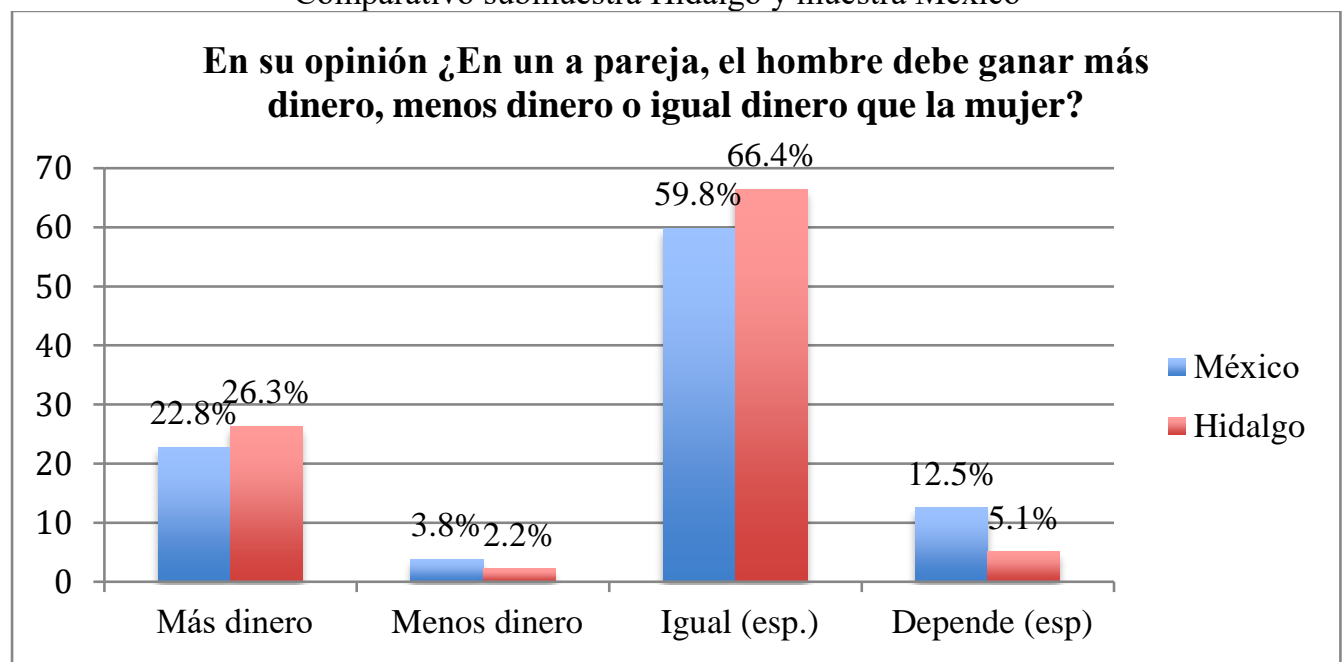

Fuente: Elaboración propia con base en ENADIS 2010.

Si bien, en más de seis puntos porcentuales la Hidalguenses señalan como ideal que el salario se equitativo, a diferencia lo señalado en la muestra nacional, $26.3 \%$ de mujeres lo refirieron como preferencia a que sean los hombres quienes deben de ganar más dinero que ellas.

Tanto es así, que incluso se considera que cuando sucede, se le pierde respeto a los hombres. Esta idea es coincidente con $35 \%$ de mujeres Hidalguenses encuestadas y en diez puntos porcentuales del promedio nacional (Gráfico 8)

Gráfico 8. Porcentaje percepción de falta de respeto al hombre por ingresos superiores de mujeres. Comparativo submuestra Hidalgo y muestra México

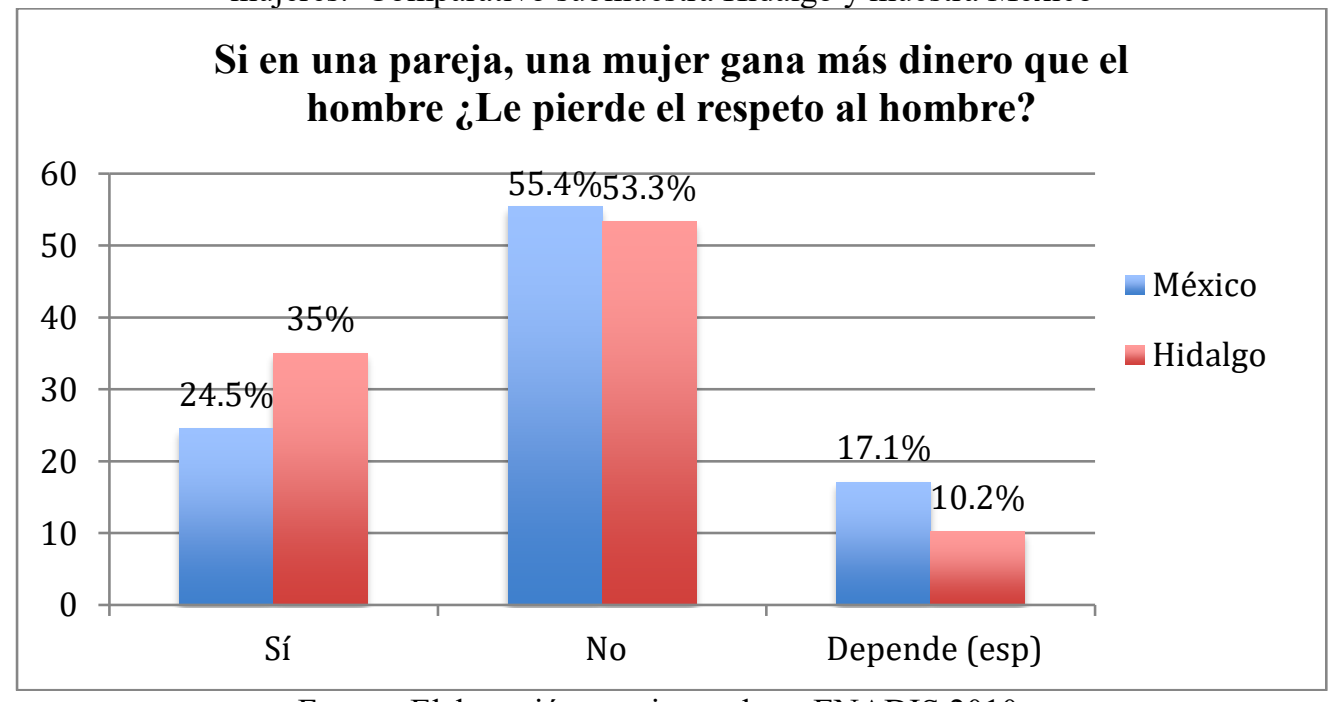

Fuente: Elaboración propia con base ENADIS 2010. 
Por otra parte, la impresión de desconocimiento y escasa empatía con los problemas particulares de las mujeres es ampliamente visible en las mujeres de esta entidad. Ya que superan considerablemente a las mujeres de la encuesta general (Gráfico 9).

Gráfico 9. Porcentaje percepción de desconocimiento de problemáticas de mujeres.

Comparativo submuestra Hidalgo y muestra México

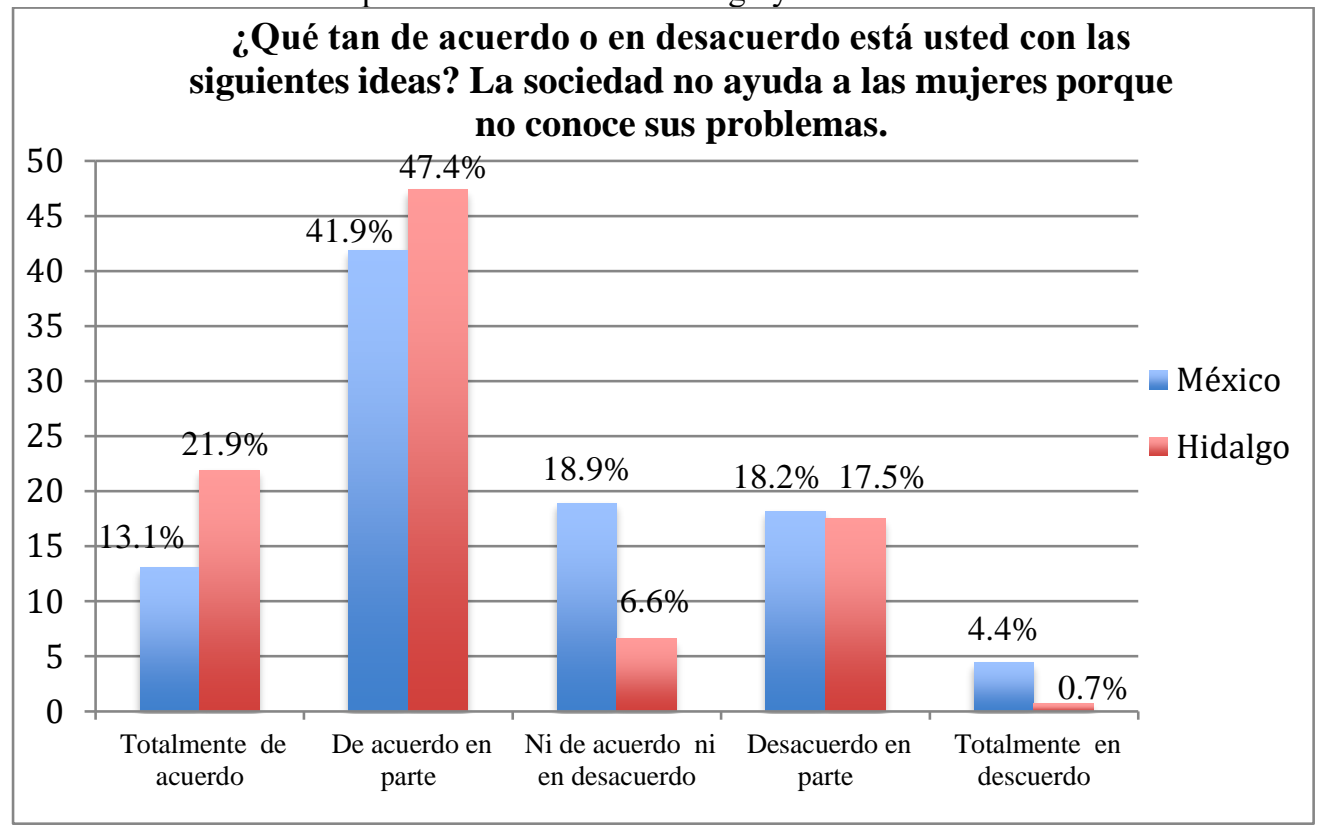

Fuente: Elaboración propia con base ENADIS 2010.

El $21.9 \%$ de las Hidalguenses considera que no se conocen sus problemas y $47.4 \%$ lo considera parcialmente. La diferencia que hay entre estos datos de la sub-muestra con la encuesta general ratifica la sensación de exacerbada de desconocimiento y por lo tanto de tratamiento especializado de las temáticas inherentes a la exclusión de las mujeres y su estructural condición que las posiciona en desventaja en distintas esferas de la vida.

\section{Conclusión}

La discriminación en el Estado de Hidalgo es un fenómeno endémico que repercute en desigualdades e inequidad de oportunidades a sectores histórica y estructuralmente marginados social y culturalmente. Según los resultados generales de la ENADIS 2010, el Estado de Hidalgo es una de las entidades en que la práctica cultural de exclusión social, estigmatización y por lo tanto expectativa de discriminación supera considerablemente al resto de estados de la federación, siendo los sectores más vulnerados las mujeres, jóvenes, homosexuales y lesbianas (Notimex, 2011). 
Como revisamos en este documento, el caso particular de las mujeres Hidalguenses, es alarmante ya que las consideraciones puntuales de valoración y actitudes referentes a la exclusión, respeto de derechos y continuidad de estereotipos (particularmente de sumisión y dependencia) es abrumadoramente superior a diferencia del promedio nacional. A su vez, estas concepciones son reiteradas por las mismas víctimas, ya que es visible la coincidencia entre los aspectos de subvaloración con los criterios de opresión y violencia que sustentan en la encuesta. Aunque al mismo tiempo reconocen la necesidad de atender las situaciones que las aquejan y posicionan en riesgo.

Este ejercicio pretendió aproximarse, de forma sucinta, a los resultados que la última encuesta sobre discriminación en una entidad en la que los derechos sobre el cuerpo de las mujeres se encuentran monopolizados por el Estado y por tanto es criminalizado; en una entidad que se niega a emitir alertas de género que, por ley, deben emitirse ante emergencias que ponen en riesgo la vida de las mujeres; como lo acontecido en el municipio de Tula en 2013 (Rincón, 2013), situación que no ha sido atendido conforme las obligaciones jurídicas asumidas internacionalmente así como por la entidad, a causa de la declaración del Comité de Expertas del Mecanismo de Seguimiento de la Convención Belem do Pará (Chávez, 2014). Estado o entidad que de forma sistemática discrimina, criminaliza y desprotege a las mujeres en general. Situación que se agrava según la condición y situación de estas: pobreza, migración, marginalidad, etcétera.

Por último, este documento es el inicio de un proyecto de investigación que pretende profundizar en el fenómeno discriminatorio de la entidad mediante diversas técnicas predictivas de corte cuantitativo para explicar y de corte interpretativo para comprender el fenómeno.

\section{References:}

1. Araiza, C. (2005). La discriminación en México: una mirada desde el análisis de las políticas públicas. El Cotidiano, (134) 30-37. Obtenido de http://www.redalyc.org/articulo.oa?id=32513405

2. Barba, C. (2012). Encuesta Nacional sobre discriminación 2010. Espiral 19(54). Pp: 261-270. Obtenido de: http://www.scielo.org.mx/scielo.php?script=sci_arttext\&pid=S166505652012000200011

3. Bourdieu, P. (2005). La dominación masculina. Barcelona: Anagrama.

4. Bucio R. \& Fix-Fierro, H. (2011). Encuesta Nacional sobre Discriminación. ENADIS 2010. Resultados Generales. México: Consejo Nacional para Prevenir la Discriminación. Obtenido de: 
http://www.conapred.org.mx/userfiles/files/Enadis-2010-RG-Accss002.pdf

5. Caïs, J. (2002). Metodología del análisis comparativo. Madrid: Centro Investigaciones Sociológicas.

6. Colino, C. (2009). "Método Comparativo", en Diccionario Crítico de Ciencias Sociales. Terminología científico - social, Reyes Román, Dir. Madrid: Plaza y Valdes \& Universidad Complutense de Madrid. Obtenido de: http://pendientedemigracion.ucm.es/info/eurotheo/diccionario/M/met odocomparativo_a.htm

7. Chavez, A. (14 de mayo de 2014). Inauguran Convención Belem do Pará en Pachuca. Milenio diario. Obtenido de: http://www.milenio.com/estados/desigualdad-foro_de_igualdadmujeres-Hidalgo_0_298770322.html

8. Consejo Nacional para Prevenir la Discriminación (2011). Encuesta Nacional sobre Discriminación en México/ Enadis 2010. Obtenido de:

http://www.conapred.org.mx/index.php?contenido=pagina\&id=424\& id_opcion=436\&op $=436$

9. Constitución Política de los Estados Unidos Mexicanos (2016). México.

10. Convención sobre la Eliminación de todas las Formas de Discriminación contra la Mujer, CEDAW. (2011). México: Organización de las Naciones Unidas.

11. Durkheim, E. (2001). Las reglas del método sociológico. México: Fondo de Cultura Económica.

12. Fernández, P. (2014) Dinámica demográfica 19902010 y proyecciones de población 2010-2030. Hidalgo. México: Consejo Nacional de Población. Obtenido de: http://www.conapo.gob.mx/work/models/CONAPO/Proyecciones/Cu adernos/13_Cuadernillo_Hidalgo.pdf

13. González, M. (1989). La mujer y la reivindicación internacional de sus derechos. México: UAM Azcapotzalco.

14. Kohn, M. (1987). "Cross-national research as an analytic strategy: American Sociological Association, 1987. Presidental Adress" American Sociological Review 52(6). pp:713-731. Obtenido de: http://www.jstor.org/stable/2095831.

15. Lagarde, M. (2012). Los cautiverios de las mujeres. Madresposas, monjas, putas, presas y locas. México: UNAM.

16. Ley Federal para Prevenir y Erradicar la Discriminación (2003). Secretaría de Gobernación. Consejo Nacional para Prevenir la 
Discriminación.

México.

Obtenido

de: http://www.conapred.org.mx/userfiles/files/LFPED_web_ACCSS.pdf

17. Mills, M., Van de Bunt, G., \& De Brujin, J. (2006). Comparative Research. Persistent problems and promising solutions. International Sociology 21(5). Pp:

619-631. https://doi.org/10.1177/0268580906067833

18. NOTIMEX (12 de julio 2011). Presentan encuesta sobre discriminación en Hidalgo. SDP Noticias. Obtenido de: https://www.sdpnoticias.com/notas/2011/07/12/presentan-encuestasobre-discriminacion-en-hidalgo

19. Oakley, A. (1977). La mujer discriminada. Biología y sociedad. Madrid: Debate.

20. Pennings, P., Keman, H., \& Kleinnijenhuis, J. (2006). Doing research in political science. An introduction to comparative methods and statistics. London: SAGE Publications.

21. Ragin, C. \& Zaret, D. (1983). Theory and Method in comparative research: two strategies. Social Forces 61(3). Pp: 731-754. doi: $10.2307 / 2578132$

22. Rihoux, B. (2006). Qualitative Comparative Analysis (QCA) and related systematic comparative methods and remaining challenges for social sciences research. International Sociology 21(5). Pp:679-706. Obtenido de: https://doi.org/10.1177/0268580906067836

23. Rincón, E. (28 de enero de 2013). Marchan cientos para frenar feminicidios en Tula , Hidalgo. Excelsior. Obtenido de: http://www.excelsior.com.mx/2013/01/28/nacional/881438

24. Rincón, G. (2005). Rasgos y retos de la lucha contra la discriminación en México. El Cotidiano, (134) 7-11. Recuperado de http://www.redalyc.org/articulo.oa?id=32513402

25. Rodríguez, L. (2011). El método comparado y la teoría de sistemas complejos. Hacia la apertura de un dialogo interdisciplinario entre la ciencia política y las ciencias de la complejidad. Reflexión Política 13(25). Pp: 78-92. Recuperado de: http://www.redalyc.org/articulo.oa?id=11018897007

26. Rodríguez Zepeda, J. (2005). Definición y concepto de la no discriminación. El Cotidiano, (134). Pp: 23-29. Recuperado de http://www.uacm.kirj.redalyc.redalyc.org/articulo.oa?id=32513404

27. Rubín, G. (2003). El tráfico de mujeres. Economía política del sexo. en Marta Lamas (Coomp.). El género. Construcción cultural de la diferencia sexual. México: Miguel Ángel Porrúa y Programa Universitario de Estudios de Género-UNAM. 
28. Sartori, G. (1970). Concept Misformation in Comparative Politics. The American Political Science Review. 64(4). 1033-1053. doi: $10.2307 / 1958356$

29. Serret, E. (2001). El género y lo simbólico. La constitución imaginaria de la identidad femenina. México: UAM-A.

30. Vigour, C. (2011). Comparision: A fundacional Approach in the social sciences. En

31. Queúdraogo, J. \& Cardoso, C. (Edit's). Readings in Methodology. African Perspectives. (pp: 215-248). Senegal: Council for the Development of Social Sciences Research in Africa (CODERSIA). Recuperado de http://muse.jhu.edu/books/9782869785175 